\title{
Trabeculectomy with mitomycin C for post-keratoplasty glaucoma
}

Misaki Ishioka, Jun Shimazaki, Junkichi Yamagami, Hiroshi Fujishima, Shigeto Shimmura, Kazuo Tsubota

\begin{abstract}
Aim-To investigate the effect of trabeculectomy with and without mitomycin $\mathrm{C}$ in post-keratoplasty glaucoma.

Methods-A retrospective study was performed on patients who underwent trabeculectomy for glaucoma after penetrating keratoplasty. 34 eyes of 32 patients were included in this study. 26 eyes received trabeculectomy with mitomycin $C$ and eight eyes without mitomycin C. The procedure was deemed successful if the intraocular pressure was maintained below $21 \mathrm{~mm} \mathrm{Hg}$ with or without use of additional antiglaucoma medication (mean follow up time 22.3 (SD 10.3) months).
\end{abstract}

Results-At the last examination trabeculectomy was successful in 19 of 26 eyes $(73.0 \%)$ with mitomycin $\mathrm{C}(+)$ and two of eight $(25.0 \%)$ without $(p=0.0219)$. When the prognosis was analysed by KaplanMeier curve, the mitomycin C (+) group showed a better prognosis $(p=0.0182)$. Mean intraocular pressure and average number of glaucoma medications improved in the group with mitomycin C without severe side effects on the graft. Graft rejection after trabeculectomy was seen in two eyes in the mitomycin C group. Final graft clarity rate was $69.2 \%$ $(18 / 26)$ in the mitomycin C (+) group and $37.5 \%(3 / 8)$ in the mitomycin C (-) group. Complications such as persistent epithelial defect, cystoid macular oedema, choroidal detachment, leakage from bleb were seen in four eyes in the mitomycin $C$ (+) group and in one eye in the mitomycin C (-) group.

Department of Ophthalmology, Tokyo Dental College, Japan M Ishioka

J Shimazaki

H Fujishima

S Shimmura

K Tsubota

Department of Ophthalmology, JR Tokyo General Hospital, Japan J Yamagami

Correspondence to: Kazuo Tsubota, MD, Department of

Ophthalmology, Tokyo

Dental College, 5-11-13

Sugano Ichikawa-shi Chiba,

Japan 272-8513

kazuo@eyebank.or.jp

Accepted for publication 24 February 2000

Elevation of intraocular pressure following penetrating keratoplasty is a serious clinical complication. According to previous reports, the incidence of glaucoma is $12-18 \%$ after penetrating keratoplasty. ${ }^{12}$ The difficulty with glaucoma surgery after keratoplasty is to perform the operation with minimal adverse effects on the corneal graft. Mitomycin C is an antiproliferative agent which is used to improve the filtration effect of trabeculectomy. ${ }^{3}$ The efficacy and safety of trabeculectomy with mitomycin $\mathrm{C}$ after penetrating keratoplasty is still not clear.
In this report, we investigated the results of trabeculectomy for post-keratoplasty glaucoma, and evaluated the effect of mitomycin C.

\section{Methods}

PATIENTS

A chart review was made of all patients who underwent trabeculectomy for glaucoma after penetrating keratoplasty at the department of ophthalmology, Tokyo Dental College between 1992 and 1997. The following information was documented for each patient: age, sex, visual acuity, intraocular pressure, diagnosis, presence of glaucoma before keratoplasty, medications used, lens status, graft status after trabeculectomy, complications after trabeculectomy, and additional treatment or operations required after trabeculectomy. Eyes which were followed for more than 6 months were enrolled in this study. Two surgeons were involved in this study.

Thirty four eyes of 32 patients were included in the study. If both eyes were included, each eye was evaluated independently. The mean age was 58.0 (SD 16.8, range 22-85) years. There were 19 men and 13 women. There was no deviation of original disease, operation method, glaucoma status before keratoplasty, ocular pressure, number of glaucoma medications, and lens status at the time of trabeculectomy between the mitomycin $\mathrm{C}(+)$ group and mitomycin C (-) group as described in Tables 1 and 2. Types of glaucoma were as follows; 10 eyes with open angle glaucoma, 10 eyes with angle closure glaucoma and six eyes with unknown aetiology in the mitomycin C (+) group; four eyes with open angle glaucoma, one eye with angle closure glaucoma, and three eyes with unknown aetiology in the mitomycin C (-) group. Angle examination, clinical history, and information from previous doctors were considered in the diagnosis of the type of glaucoma. Certain cases after limbal transplantation did not allow a detailed evaluation of the angle. Surgeries before penetrating keratoplasty were: keratolimbal allograft transplantation (five eyes), trabeculectomy (three eyes),
Table 1 Original disorder for which penetrating keratoplasty was performed

\begin{tabular}{lll}
\hline Indication & $\begin{array}{l}\text { Mitomycin } C \\
(+)(26 \text { eyes })\end{array}$ & $\begin{array}{l}\text { Mitomycin } C \\
(-) \text { (8 eyes) }\end{array}$ \\
\hline Bullous keratopathy & 10 & 4 \\
Congenital corneal opacity & 3 & 0 \\
Perforative ulcer & 4 & 0 \\
Chemical burn & 3 & 1 \\
Leucoma & 2 & 2 \\
Keratoconus & 2 & 0 \\
Ocular cicatricial pemphigoid & 1 & 1 \\
Lattice dystrophy & 1 & 0 \\
\hline
\end{tabular}


Table 2 Preoperative status

\begin{tabular}{lll}
\hline & Mitomycin C (+) (26 eyes) & Mitomycin C (-) (8 eyes) \\
\hline Eyes with limbal transplantation & $7(26.9 \%)$ & $5(62.5 \%)$ \\
Eyes with glaucoma & $6(23.1 \%)$ & $3(37.5 \%)$ \\
Intraocular pressure (mm Hg) & $32.3(7.4)$ & $31.8(7.5)$ \\
Number of glaucoma medications & $3.2(1.0)$ & $3.3(0.9)$ \\
$\quad$ Range & $(2-5)$ & $(2-4)$ \\
Lens status & & \\
$\quad$ Phakia & 2 eyes $(7.7 \%)$ & 0 eyes $(0 \%)$ \\
$\quad$ Aphakia & $5(19.2 \%)$ & $2(25 \%)$ \\
$\quad$ Anterior chamber lens & $4(15.4 \%)$ & $3(37.5 \%)$ \\
Posterior chamber lens & $15(57.7 \%)$ & $3(37.5 \%)$ \\
\hline
\end{tabular}

conjunctival cover for perforation (two eyes), and retinal detachment surgery (one eye). Follow up ranged from 6 to 40 months, with a mean 22.3 (10.3) months. Patients were followed even after they were evaluated as failure cases. Twenty six eyes received trabeculectomy with mitomycin $\mathrm{C}$ and eight without mitomycin C. Trabeculectomy without mitomycin C was performed in the early period of this study (from 1992 to 1994) when mitomycin C was not routinely used for postkeratoplasty glaucoma. There was no statistical difference in follow up time between the mitomycin C (+) group $(19.3(8.1))$ and the mitomycin (-) group (31.4 (10.6)) $(\mathrm{p}=0.1017$, Student's $t$ test). Twelve eyes underwent keratolimbal allograft transplantation with keratoplasty before trabeculectomy.

All eyes underwent trabeculectomy after keratoplasty. There were no eyes which received keratoplasty and trabeculectomy simultaneously.

\section{SURGICAL PROCEDURE}

After retrobulbar anaesthesia was administered, care was taken to reduce the intraocular pressure by digital massage. A superior conjunctival and Tenon's incision was performed as far posteriorly as possible, and a limbal based flap was dissected. A partial thickness square scleral flap was then dissected. For the group with mitomycin $\mathrm{C}$, a sponge soaked with a $0.04 \%$ solution of mitomycin $\mathrm{C}$ was placed on and under the scleral flap and left for 3 minutes. Subsequently, the sponge was removed and the area was washed with balanced salt solution.

After the excision of the corneoscleral block, a peripheral iridectomy was performed. The scleral flap was tightly closed with 10-0 nylon sutures. The conjunctiva and Tenon's capsule were closed with 10-0 nylon running sutures.

EVALUATION

The procedure was deemed successful if the intraocular pressure was maintained below 21 $\mathrm{mm} \mathrm{Hg}$ with or without the use of additional antiglaucoma medication. Suture lysis of the flap by argon laser or a 27 gauge needle was done when bleb filtration was insufficient. When maximum tolerable therapy was insuffi-

Table 3 Effect of trabeculectomy

\begin{tabular}{lll}
\hline & Mitomycin C (+) (26 eyes) & Mitomycin C (-) (8 eyes) \\
\hline Successful control with no medication & $13(50 \%)$ & $2(25 \%)$ \\
Successful control with topical medication & $6(23.1 \%)$ & $0(0 \%)$ \\
Unsuccessful control & $7(26.9 \%)$ & $6(75 \%)$ \\
\hline
\end{tabular}

cient to control intraocular pressure below 21 $\mathrm{mm} \mathrm{Hg}$, the case was designated as unsuccessful. Most cases in the unsuccessful group underwent further glaucoma surgery.

The preoperative intraocular pressure was determined by the average of the last three previously recorded pressures before surgery, measured with a Tonopen (Mentor O\&O, Norwell, MA, USA) or Goldmann applanation tonometer. The data of the ocular pressure and visual acuity at the last follow up were evaluated as postoperative data. If additional glaucoma surgery was necessary, the data just before this additional surgery were considered as the last follow up data.

Change in visual acuity was defined as improved when postoperative acuity gained two or more lines of spectacle corrected visual acuity with respect to the preoperative acuity; as worse when postoperative acuity lost two or more lines of spectacle corrected visual acuity; and as unchanged when postoperative acuity was kept within one line of spectacle corrected visual acuity with respect to the preoperative value.

The graft was evaluated as decompensated when endothelial function deteriorated to levels where the corneal thickness could not be maintained within normal values because of corneal oedema.

\section{Results}

EFFECT OF TRABECULECTOMY AT LAST FOLLOW UP

At the last examination, 19 of 26 eyes $(73.0 \%)$ with mitomycin $\mathrm{C}(+)$ trabeculectomy were successful, and two of eight $(25.0 \%)$ with mitomycin C (-) trabeculectomy $(\mathrm{p}=0.0219$ by Fisher's test) (Table 3). Among 19 successful eyes with mitomycin C (+) trabeculectomy, 13 were successfully controlled without any glaucoma medications and six eyes needed eye drops for ocular pressure control. When the prognosis was analysed by Kaplan-Meier curve, the mitomycin C (+) group showed better prognosis with statistical significance (Fig 1, p=0.0182) Most cases without mitomycin $\mathrm{C}$ failed within 6 months.

Among 12 eyes with keratolimbal allograft transplantation before trabeculectomy, seven eyes underwent trabeculectomy with mitomycin $\mathrm{C}$ and five eyes without mitomycin C. In

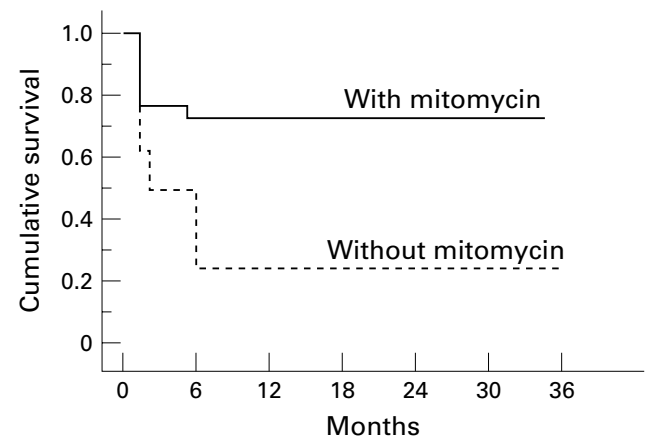

Figure 1 Probability of surgical success in trabeculectomy of post-keratoplasty glaucoma. Surgical success is defined as the maintenance of intraocular pressure below $21 \mathrm{~mm} \mathrm{Hg}$ $(p=0.0182$, Kaplan-Meier survival analysis with Mantel-Cox log rank test). 


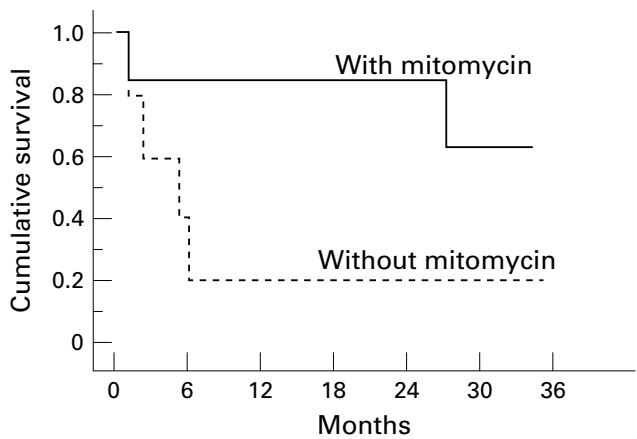

Figure 2 Probability of surgical success in trabeculectomy of post-keratoplasty glaucoma with limbal transplantation. Surgical success is defined as the maintenance of intraocular pressure below $21 \mathrm{~mm} \mathrm{Hg}(p=0.0763$, Kaplan-Meier survival analysis with Mantel-Cox log rank test).

the seven mitomycin $\mathrm{C}(+)$ eyes, five eyes were evaluated as successful whereas one of five eyes was considered successful with mitomycin C (-) trabeculectomy $(\mathrm{p}=0.1212$ by Fisher's test). Figure 2 shows the prognosis of these two groups by Kaplan-Meier curve.

\section{CHANGE IN INTRAOCULAR PRESSURE AND}

NUMBER OF GLAUCOMA MEDICATION

Mean intraocular pressure lowered from 32.3 (7.4) $\mathrm{mm} \mathrm{Hg}$ to 21.1 (13.5) $\mathrm{mm} \mathrm{Hg}$ in eyes with mitomycin $\mathrm{C}(\mathrm{p}=0.0002)(14.4(3.5) \mathrm{mm}$ $\mathrm{Hg}$ in the successful eyes with mitomycin $\mathrm{C})$, and from 31.8 (7.5) to 24.8 (8.0) $\mathrm{mm} \mathrm{Hg}$ in the group without mitomycin $\mathrm{C}(\mathrm{p}=0.017)$. There was a statistical difference in postoperative intraocular pressure $(\mathrm{p}=0.0034)$ between the mitomycin $\mathrm{C}(+)$ group and mitomycin $\mathrm{C}$ (-) group.

The average number of glaucoma medications was $3.2(1.0)$ with a range of $2-5$ used before trabeculectomy and 1.5 (1.7) with a range of $0-5$ used after trabeculectomy in the group with mitomycin $\mathrm{C}(\mathrm{p}=0.0001)$. These numbers were $3.3(0.9)$ with a range of $2-4$ and 2.6 (1.7) with a range of $0-4$ in the group without mitomycin $C(p=0.095)$. When comparing the medication number after trabeculectomy, the number was less in the mitomycin $\mathrm{C}(+)$ group $(\mathrm{p}=0.0185)$.

\section{VISUAL ACUITY}

Table 4 shows the change in visual acuity. Reasons for decreased visual acuity were: bullous keratoplasty (five eyes), retinal detachment (one eye), corneal conjunctivalisation (one eye), and membrane formation on intraocular lens (one eye) in the mitomycin C (+) group; and glaucoma (three eyes) and irregular astigmatism (one eye) in the mitomycin C (-) group. There was no significant

Table 4 Postoperative status

\begin{tabular}{lll}
\hline & Mitomycin $C(+)$ 26 eyes & Mitomycin $C(-)$ 8 eyes \\
\hline Intraocular pressure $(\mathrm{mm} \mathrm{Hg})$ & $21.1(13.5)$ & $24.8(8.0)$ \\
Number of glaucoma medications & $1.5(1.7)$ & $2.6(1.7)$ \\
$\quad$ Range & $(0-5)$ & $(0-4)$ \\
Clear graft & $18(69.2 \%)$ & $3(37.5 \%)$ \\
Visual acuity & & \\
$\quad$ Improved & $6(23.1 \%)$ & $0(0 \%)$ \\
$\quad$ Same & $12(46.1 \%)$ & $4(50 \%)$ \\
$\quad$ Worse & $8(30.8 \%)$ & $4(50 \%)$ \\
\hline
\end{tabular}

Table 5 Complications of trabeculectomy

\begin{tabular}{lll}
\hline & $\begin{array}{l}\text { Mitomycin C (+) } \\
(26 \text { eyes) }\end{array}$ & $\begin{array}{l}\text { Mitomycin } C \\
(-)(8 \text { eyes })\end{array}$ \\
\hline $\begin{array}{l}\text { Persistent epithelial defect } \\
\text { Cystoid macular oedema }\end{array}$ & $1(3.8 \%)$ & $1(12.5 \%)$ \\
$\begin{array}{l}\text { Choroidal detachment } \\
\text { Leakage from bleb }\end{array}$ & $1(3.8 \%)$ & $0(0 \%)$ \\
$\begin{array}{l}\text { Rejection } \\
\quad \text { (reversible) }\end{array}$ & $1(3.8 \%)$ & $0(0 \%)$ \\
$\quad$ irreversible) & $1(3.8 \%)$ & $0(0 \%)$ \\
\begin{tabular}{l} 
Graft decompensation \\
\hline
\end{tabular} & $1(3.8 \%)$ & $0(0 \%)$ \\
\hline
\end{tabular}

difference in postoperative visual acuity between mitomycin $\mathrm{C}(+)$ group and the mitomycin $\mathrm{C}(-)$ group.

GRAFT REJECTION AND FINAL GRAFT CLARITY After trabeculectomy, two eyes in the group with mitomycin C experienced corneal endothelial graft rejection. These episodes occurred 4 months after surgery. One cornea cleared with systemic corticosteroid administration, but the other eye developed graft endothelial decompensation. Five eyes in the mitomycin $\mathrm{C}(+)$ group and one eye in the mitomycin C (-) group experienced decompensation without a history of graft rejection. Two eyes in the mitomycin $\mathrm{C}(+)$ group and four eyes in the mitomycin $\mathrm{C}(-)$ group experienced decompensation after additional surgery-retinal detachment surgery for one eye in the mitomycin $\mathrm{C}(+)$ group and glaucoma surgeries for others. Final graft clarity rate was $69.2 \%(18 / 26)$ in the mitomycin C $(+)$ group and $37.5 \%(3 / 8)$ in the mitomycin C (-) group.

\section{COMPLICATIONS}

The complications of trabeculectomy were seen in unsuccessfully controlled eyes (Table 5). Persistent epithelial defect appeared in two eyes - one in the mitomycin $\mathrm{C}(+)$ group and one in the mitomycin $\mathrm{C}(-)$ group. The former eye had limbal deficiency after chemical burn, and required several limbal transplantations, but suffered from a recurrent limbal deficiency. Cystoid macular oedema and choroidal detachment were seen in the mitomycin C ( + ) group. A leaking bleb was seen in one eye with mitomycin $\mathrm{C}(+)$ trabeculectomy after digital massage, but without any complications or additional surgery.

ADDITIONAL TREATMENT IN THE UNSUCCESSFUL GROUP

Six of seven eyes in which mitomycin C (+) trabeculectomy alone was insufficient to control intraocular pressure, were successfully controlled with additional 5-fluorouracil injection (three eyes), Nd:YAG cyclophotocoagulation (two eyes), vitrectomy (one eye). 5-Fluorouracil was injected near the bleb following bleb needling or revision. The reason for vitrectomy was that the vitreous herniated in the trabeculectomy window. Graft decompensation was seen after Nd:YAG cyclophotocoagulation. Among six unsuccessfully controlled eyes in the mitomycin C (-) group, only one eye was successfully controlled with Nd:YAG cyclophotocoagulation. Two eyes did 
not receive any additional surgery because their visual acuity were hand movements or no light perception. Three eyes underwent other glaucoma surgeries (another trabeculectomy, sinusotomy, shunt operation, Nd:YAG cyclophotocoagulation) but resulted in unsuccessful intraocular pressure control or phthisis.

\section{Discussion}

The results of glaucoma surgery are inconsistent after penetrating keratoplasty. Sufficient reduction of ocular pressure is necessary; however, the procedure should be minimally invasive to the corneal graft. Simple trabeculectomy is a popular method, but with a low success rate $(70 \%$ successful control at 9 months and $50 \%$ by 2 years for postkeratoplasty glaucoma ${ }^{4}$ ). Cyclocryotherapy and cyclophotocoagulation with Nd:YAG yields a high success rate $^{5-7}$ but induces more complications, some of which are severe such as phthisis bulbi. Shunt operation is another choice of glaucoma operation after penetrating keratoplasty, but this operation showed high rate of graft failure. ${ }^{89}$

Antiproliferative agents such as 5-fluorouracil or mitomycin C are used to improve the filtration effect of trabeculectomy. Mitomycin C is more effective in controlling ocular pressure compared with 5-fluorouracil. More complications including corneal epithelial defect were seen after trabeculectomy with 5-fluorouracil. ${ }^{3}$ In this report, our results show that trabeculectomy with mitomycin C achieved a statistically lower ocular pressure compared with trabeculectomy without mitomycin $\mathrm{C}$. The number of glaucoma medication required were also lower when mitomycin $\mathrm{C}$ was used. Kaplan-Meier analysis revealed that trabeculectomy with mitomycin C survived longer than trabeculectomy without mitomycin C, with statistical significance. Most cases without mitomycin $\mathrm{C}$ failed within 6 months.

Simultaneous trabeculectomy and keratoplasty is another option for patients with a history of uncontrolled glaucoma at the time of keratoplasty surgery. Although a single procedure may be less stressful for the donor graft, a recent report has shown that a combined procedure has a lower chance of long term intraocular pressure control. ${ }^{10}$

Compared with previous reports of cyclocryotherapy and cyclophotocoagulation with Nd:YAG, complications associated with mitomycin C trabeculectomy seem to be less severe. Moreover, after trabeculectomy with mitomy- cin C, additional surgery or treatment was effective in unsuccessfully controlled eyes with no complications, whereas eyes with trabeculectomy without mitomycin $\mathrm{C}$ had a poor prognosis. The graft clarity rate was better in mitomycin $C(+)$ group. It seems likely that mitomycin $\mathrm{C}$ has a positive adjuvant effect on trabeculectomy without increasing the risk of graft failure.

Limbal transplantation is a method to reconstruct the corneal epithelium for the cases with limbal deficiency, ${ }^{11}$ and is sometimes performed simultaneously with penetrating keratoplasty. Among the patients with limbal transplantation, the mitomycin C (+) group showed better results in the analysis by Kaplan-Meier curve (Fig 2), but the number of patients were too small to observe statistical differences. Further analysis is necessary to see the effect of mitomycin $\mathrm{C}$ in trabeculectomy following limbal transplantation.

Trabeculectomy with mitomycin C showed satisfactory results following keratoplasty and keratoplasty combined with limbal transplantation. When we experience difficulty in controlling ocular pressure following keratoplasty, simple trabeculectomy should not really be a choice unless a major contraindication to mitomycin $\mathrm{C}$ exists.

Proprietary interest: None

1 Foulks GN. Glaucoma associated with penetrating keratoplasty. Ophthalmology 1987;94:871-4

2 Chien AM, Schmidt AM, Cohen EJ, et al. Glaucoma in the mmediate postoperative period after penetrating keratoplasty. Am f Ophthalmol 1993;115:711-14

3 Kitazawa Y, Kawase K, Matsushita H, et al. Trabeculectomy with mitomycin C. A comparative study with fluorouracil. Arch Ophthalmol 1991;109:1693-8.

4 Gilvarry AME, Kirkness CM, Steele ADM, et al. The management of post-keratoplasty glaucoma by trabeculectomy. Eye 1989;3:717-18.

5 Binder PS, Abel R, Kaufman HE. Cyclocryotherapy for glaucoma after penetrating keratoplasty. Am f Ophthalmol 1975;79:489-92.

6 West CE, Wood TO, Kaufman HE. Cyclocryotherapy for glaucoma pre- or postpenetrating keratoplasty. $A m$ F Ophthalmol 1973;76:485-9.

7 Threlkeld AB, Shields MB. Noncontact transscleral Nd: YAG cyclophotocoagulation for glaucoma after penetrating keratoplasty. Am f Ophthalmol 1995;120:569-76.

8 McDonnell PJ, Robin JB, Schanzlin DJ, et al. Molteno implant for control of glaucoma in eyes after penetrating keratoplasty. Ophthalmology 1988;95:364-9.

9 Beebe WE, Starita RJ, Fellman RL, et al. The use of Molteno implant and anterior chamber tube shunt to encircling band for the treatment of glaucoma in keratoplasty patients. Ophthalmology 1990;97:1414-22

10 WuDunn D, Alfonso E, Palmberg PF. Combined penetrating keratoplasty and trabeculectomy with mitomycin $\mathrm{C}$. Ophthalmology 1999;106:396-400.

11 Tsubota K, Satake Y, Ohyama M, et al. Surgical reconstruction of the ocular surface in advanced ocular cicatricial pemphigoid and Stevens-Johnson syndrome. Am $₹ \mathrm{Oph}$ thalmol 1996;122:38-52. 